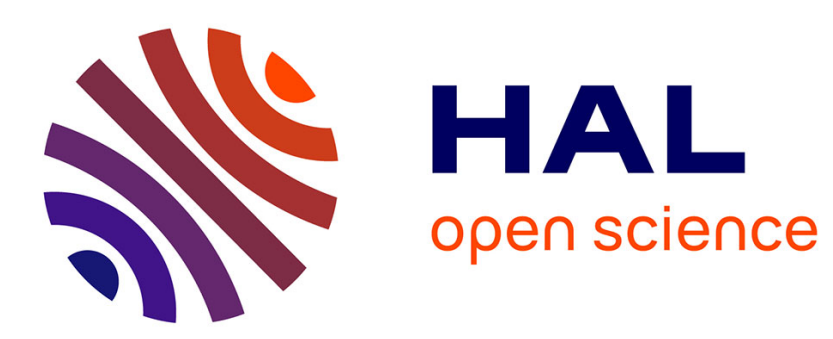

\title{
MoM and PEEC Method to Reach a Complete Equivalent Circuit of a Static Converter
}

Vincent Ardon, Jérémie Aimé, Olivier Chadebec, Edith Clavel, Enrico

Vialardi

\section{- To cite this version:}

Vincent Ardon, Jérémie Aimé, Olivier Chadebec, Edith Clavel, Enrico Vialardi. MoM and PEEC Method to Reach a Complete Equivalent Circuit of a Static Converter. EMC Zurich, Jan 2009, Zurich, Switzerland. hal-00354293

\section{HAL Id: hal-00354293 https://hal.science/hal-00354293}

Submitted on 19 Jan 2009

HAL is a multi-disciplinary open access archive for the deposit and dissemination of scientific research documents, whether they are published or not. The documents may come from teaching and research institutions in France or abroad, or from public or private research centers.
L'archive ouverte pluridisciplinaire $\mathbf{H A L}$, est destinée au dépôt et à la diffusion de documents scientifiques de niveau recherche, publiés ou non, émanant des établissements d'enseignement et de recherche français ou étrangers, des laboratoires publics ou privés. 


\title{
MoM and PEEC Method to Reach a Complete Equivalent Circuit of a Static Converter
}

\author{
Vincent Ardon ${ }^{\# * 1}$, Jérémie Aimé ${ }^{\# 2}$, Olivier Chadebec ${ }^{\# 3}$, Edith Clavel ${ }^{\# 4}$, Enrico Vialardi ${ }^{* 5}$

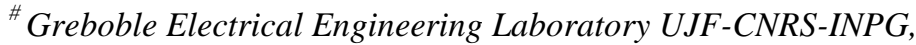 \\ BP 4638402 Saint-Martin-d'Hères Cedex - France \\ ${ }^{1}$ ardon.vincenteg2elab.inpg.fr \\ 2 jeremie.aimedg2elab.inpg.fr \\ ${ }^{3}$ olivier.chadebec@g2elab.inpg. fr \\ ${ }^{4}$ edith.clavel@g2elab.inpg.fr \\ * Cedrat \\ 15 rue de Malacher, 38246 Meylan Cedex - France \\ ${ }^{5}$ enrico.vialardi@cedrat.com \\ Schneider Electric \\ Corporate Research Center Science \& Technology Schneider Electric, 37 quai P.-L. Merlin 38050 Grenoble - France
}

\begin{abstract}
This paper presents an application of the Method of Moments (MoM), to compute parasitic capacitances of a power electronics layout. By coupling these capacitances to a resistive and inductive equivalent circuit obtained thanks to Partial Element Equivalent Circuit (PEEC) method, a complete electrical equivalent circuit made of lumped elements is obtained. Its simulation by means of a SPICE-like tool enables the evaluation of the EMC efficiency of a power electronics structure.

This method is applied to a boost converter and the results obtained by numerical simulation (MoM and finite element method - FEM) are compared to measurements. Finally, radiated EMC investigations are presented thanks to circuit simulation and measurements.
\end{abstract}

\section{INTRODUCTION}

Up to recent times modelling power electronic layouts with a simplified resistive and inductive PEEC model was sufficient to characterise the equivalent impedances or current distribution. In fact, to analyse the EM interactions between the power and the drive subcircuits, the capacitive effects could be neglected without degrading too much the accuracy of the model. Nowadays, this is no longer possible as power electronic systems are getting always smaller and the frequency ranges are rising. Besides, EMC considerations are now integrated early in the design stage and as a consequence, the capacitive couplings cannot be neglected anymore.

In the following section, a brief state of the art of the techniques used to evaluate the parasitic elements is presented. Then, the interest is focused on classical methods, and especially the MoM, able to systematically extract parasitic capacitances. Section IV presents the combination of the PEEC method with a MoM model applied to a commonlyused power electronics structures: a static converter. Measurements are made to validate the complete model thus obtained. Last section presents how this global equivalent circuit can be then used to evaluate the efficiency of the whole structure regarding EMC performances.

\section{StATE OF THE ART}

PEEC method is well known for being perfectly adapted to the modeling of cabling in power electronics structures [1-3]. The method has been implemented in a commerciallyavailable software [4], which models 3D-layouts by an equivalent circuit made of resistances, inductances and mutual couplings evaluated thanks to analytical formulations.

For power electronics applications where the frequencies are not so high - a few hundreds of $\mathrm{MHz}$ - and the structures are complex, this model is mostly sufficient and, if necessary, it is still possible to add to the R-L-M model the values of capacitances measured or calculated [1]. But for EMC considerations where frequency ranges are much more extended, this approach is not satisfying. Inductive couplings are considered but the stray capacitances and the capacitive couplings of the layout which are required for the determination of the common mode are missing. To this goal, a systematic technique has to be implemented.

But the calculation of the capacitive elements is not as easy as for inductances. The limits of the existing analytical formulations based on simple geometries are rapidly reached with real power electronics structures. And the question of positioning these capacitances at the right place enhances the complexity of the problem. In fact, this is usually done by inserting a capacitance where there is a high $\mathrm{dV} / \mathrm{dt}$ but this method can hardly be generalized and is often not sufficient enough. The purpose of this work is to obtain an R-L-M-C model of any cabling structure by combining well known methods with a systematic approach..

\section{CAPACITANCE EVALUATION METHODS}

\section{A. Multiconductor transmission line (MTL) equations}

Since the PEEC method provides the inductance matrix [L], one could imagine obtaining the capacitance values by matrix inversion of the simple formula $[\mathrm{L}][\mathrm{C}]=\mu \varepsilon$, as usually done for 
the computation of the p.u.l. capacitance of multiconductor transmission line [5]. Unfortunately the assumptions that have to be made to apply this equation are too restrictive for power electronics applications [6]. In fact, this firstly requires the exact knowledge of the relative permittivity \&r and secondly to have a homogeneous media. As it is not always possible to reduce the problem to an effective er permittivity a numerical approach is preferred.

\section{B. Method of Moments (MoM)}

The Method of Moments (MoM) is known as a powerful approach to model electrotechnical devices in which air (or equivalent medium) is predominant [7]. Unlike another wellknown method that is not presented here, Finite Element Method (FEM) [8], only the active materials are meshed (conductors, dielectrics) in surface elements.

To compute the capacitances between the regions composing the structure, an equivalent charge formulation is used. Free-charges are represented over the conductor and dielectric surfaces by assuming that they are ideal and embedded in a piecewise-constant medium. Each surface is meshed with $\mathrm{n}$ uniformly-distributed rectangular surface elements. Thus each of the $\mathrm{n}$ surface elements produces a potential $\mathrm{V}_{\mathrm{k}}$ given by (1) where $\mathrm{S}_{\mathrm{i}}$ is the conductor-dielectric or dielectric-dielectric interface surface $\mathrm{i}$ of charge $\mathrm{Q}_{\mathrm{i}}$, and $\mathbf{r}_{\mathrm{ki}}$ is the distance between the barycentre of the surface elements $\mathrm{k}$ and $\mathrm{i}$.

$$
V_{k}=\frac{1}{4 \pi \varepsilon_{0}} \sum_{i=1}^{n} \frac{Q_{i}}{S_{i}} \iint_{S_{i}} \frac{1}{\left\|\vec{r}_{k i}\right\|} d S_{i}
$$

This surface integral can be computed thanks to an integration technique based on Gauss points. This surface integral is numerically divergent when we compute the potential produced by a surface element on itself at this barycentre because $\mathbf{r}_{\mathrm{ii}}$ tends to zero. In order to improve the accuracy of this potential computation, an analytic formulation is used [9].

Moreover, at the dielectric-dielectric interface surface $\mathrm{k}$, the jump of the derivative of the potential produces (2) where $\varepsilon_{\mathrm{r} 1}$ and $\varepsilon_{\mathrm{r} 2}$ are the relative permittivities of the two media [10].

$$
0=-\frac{\varepsilon_{r 1}+\varepsilon_{r 2}}{2 \varepsilon_{0}\left(\varepsilon_{r 1}-\varepsilon_{r 2}\right)} \cdot \frac{Q_{k}}{S_{k}}+\frac{1}{4 \pi \varepsilon_{0}} \sum_{i=1}^{n} \frac{Q_{i}}{S_{i}} \iint_{S} \frac{\vec{r}_{k i} \cdot \vec{n}_{i}}{\left\|\overrightarrow{r_{i i}}\right\|^{3}} d S_{i}
$$

Let us consider there are $\mathrm{n}_{\mathrm{c}}$ and $\mathrm{n}_{\mathrm{d}}$ respectively conductordielectric and dielectric-dielectric interface surface elements. Consequently, $\mathrm{n}_{\mathrm{c}}+\mathrm{n}_{\mathrm{d}}=\mathrm{n}$. Thanks to a point matching approach, the two previous relations (1-2) link together the potentials and the charges of each conductor-dielectric or dielectric-dielectric interface surface element by a linear relation (3) where the coefficients of the matrix $\mathrm{P} / \mathrm{E}$ are computed with a charge of one Coulomb by surface element [11].

The square matrix $\mathrm{P} / \mathrm{E}$, modelling the interaction between each conductor - or dielectric - surface element, is necessarily dense. So, the distribution of charges is obtained by solving this linear system with a LU-decomposition.

$$
\left[\begin{array}{ccc}
P_{1,1} & \ldots & P_{1, n} \\
\vdots & & \vdots \\
P_{n c 1} & \ldots & P_{n c, n} \\
E_{n c+1,1} & \ldots & E_{n c+1, n} \\
\vdots & & \vdots \\
E_{n, 1} & \ldots & E_{n, n}
\end{array}\right] .\left[\begin{array}{c}
Q_{1} \\
\vdots \\
Q_{n c} \\
Q_{n c+1} \\
\vdots \\
Q_{n}
\end{array}\right]=\left[\begin{array}{c}
V_{1} \\
\vdots \\
V_{n c} \\
0 \\
\vdots \\
0
\end{array}\right]
$$

Algorithms based on fast multipole method (FMM) are widely used to model structures at very high frequency and which take into account the propagation phenomenon with little memory and quick integration and resolution time. But it appears that they do not converge any times for modeling this kind of complex structures (very thin and multi-layer conductors laid on dielectrics) with lower frequencies. So we propose a robust and vectorized algorithm to increase the speed of integration time. And, in order to minimize consumption of memory, an optimized mesh is used (Fig. 3b).

Computing the capacitance matrix $\mathrm{C}$ of the $\mathrm{m}$ conductor regions, and which is therefore of $\mathrm{m}^{2}$ size, requires to solve $\mathrm{m}$ times the relation (3) in the following conditions: the $j$ th column of matrix $\mathrm{C}$ is obtained for the $j$ th conductor region whose potential is set to 1 Volt and 0 Volt otherwise, and the $i$ th element of that $j$ th column is the sum of the charges of the $i$ th conductor region (4).

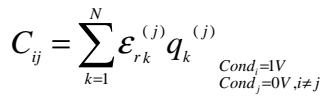

The term $\varepsilon_{r k}^{(j)}$ takes into account the nature of the interface with a dielectric medium and applies 1 , if it is air and $\varepsilon_{r}$ (or $\left.\left(\varepsilon_{r}+1\right) / 2[5]\right)$, if it is a dielectric material and the conductor is a volume (or a surface).

\section{APPLICATION TO A STATIC CONVERTER}

\section{A. Studied structure}

The system studied is a boost converter. The cabling is made on a Printed Circuit Board (PCB) and consists in $35 \mu \mathrm{m}-$ thick copper tracks placed on an epoxy layer whose thickness is $1.6 \mathrm{~mm}$. The structure is also composed of a $1.3 \mathrm{~mm}$-thick ground plane placed under the PCB, $28 \mathrm{~mm}$ far from it.

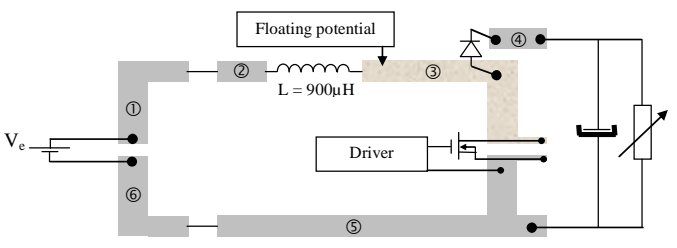

Fig. 1. Boost converter topology

Only the conductors have been modelled and the components are supposed ideal (see Fig. 1). The investigation is focused on the capacitances between conductors (coupling 
capacitances) and between the conductors and the ground plane (stray capacitances). By neglecting some couplings like those between remote conductors, twelve preponderant capacitances - six couplings, six strays- have been identified. Fig. 2 shows where these capacitances are situated.

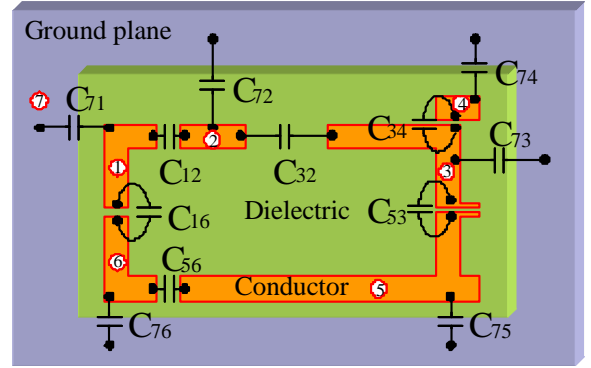

Fig. 2. Capacitances identified

\section{B. Capacitance evaluation}

Numerical simulation makes it possible to compute these values. FEM ([12]) and MoM (prototype coded in MATLAB language) methods have been used and Fig. 3 represents the meshes obtained on the studied case. As finite elements require the meshing of the surrounding air as well as an important mesh density at the edges, the number of elements is very high (about 675.000). For the MoM method the mesh is needed on the active materials only and this leads to a very much reduced mesh elements (only 6.000), and consequently, to a limited number of unknowns.
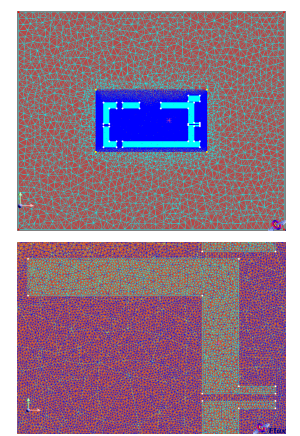

Fig. 3. a- FEM-mesh
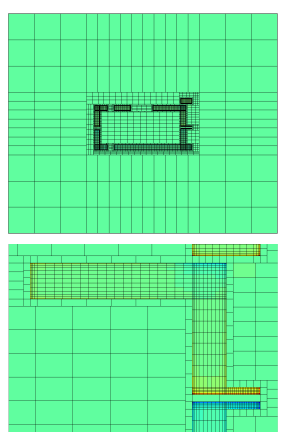

b- MoM-mesh
Besides, measurements have also been done on the boost converter [13]. It is not possible to measure directly the capacitances that have been calculated but an adapted methodology has been used to identify them. An impedance analyser is used to measure equivalent values that correspond to linear combinations of the stray and the coupling capacitances which need to be extracted. By having several measurement series the problem can be defined like a system of equations. The identification is easy and it is then possible to make a comparison with the values obtained with the FEM and MoM simulations.

The results are displayed in Table I and show a good correlation between numerical and measured capacitances values. So this comparison contributes to validate the computation of capacitances with the MoM on a real case.

TABLE I

MEASUREMENTS, MOM AND FEM CAPACITANCES

\begin{tabular}{|c|cccccc||cccccc|}
\multicolumn{1}{c|}{ Capa. (pF) } & C17 & C27 & C37 & C47 & C57 & C67 & C12 & C23 & C34 & C53 & C65 & C16 \\
\hline Mes. & 1,53 & 1,25 & 2,95 & 0,87 & 6,5 & 1,57 & 0,92 & 0,7 & 1,12 & 1,26 & 0,93 & 0,98 \\
MoM & 1,77 & 1,3 & 2,72 & 0,88 & 5,28 & 1,72 & 0,34 & 0,08 & 0,89 & 1,36 & 0,3 & 0,49 \\
FEM & 1,9 & 1,4 & 2,92 & 0,93 & 5,67 & 1,84 & 0,25 & 0,06 & 0,8 & 1,1 & $\theta, 27$ & 0,4 \\
\hline$\%$ MoM/Mes. & 15,7 & 4,0 & 7,8 & 1,1 & 18,8 & 9,6 & 63,0 & 88,6 & 20,5 & 7,9 & 59,1 & 50,0 \\
$\%$ FEM/Mes. & 24,2 & 12,0 & 1,0 & 6,9 & 12,8 & 17,2 & 72,8 & 91,4 & 28,6 & 12,7 & 71,0 & 59,2 \\
\hline
\end{tabular}

It has to be noticed that the stray capacitances $\left(\mathrm{C}_{71}, \mathrm{C}_{72}, \mathrm{C}_{73}\right.$, $\mathrm{C}_{74}, \mathrm{C}_{75}$ and $\mathrm{C}_{76}$ ) are higher than the other ones and are therefore easier to measure. On the contrary, the capacitive couplings between conductors are more difficult to measure because of their very low value. That is why there is a great difference between the numerical and measured values for $\mathrm{C}_{12}$ and $\mathrm{C}_{23}$.

\section{PEEC-MoM global model}

Previous sections have shown how it is possible to compute with a systematic approach the parasitic capacitances of a layout. Now that it is possible firstly to have reliable results thanks to numerical simulation and secondly to place these capacitances at the right place, a broadband analysis of the structure can be performed. Fig. 4 shows the implantation of capacitances between a conductor $\mathrm{i}$, defined by $\mathrm{k}$ nodes, and the ground plane. The same implantation could be done between two conductors.

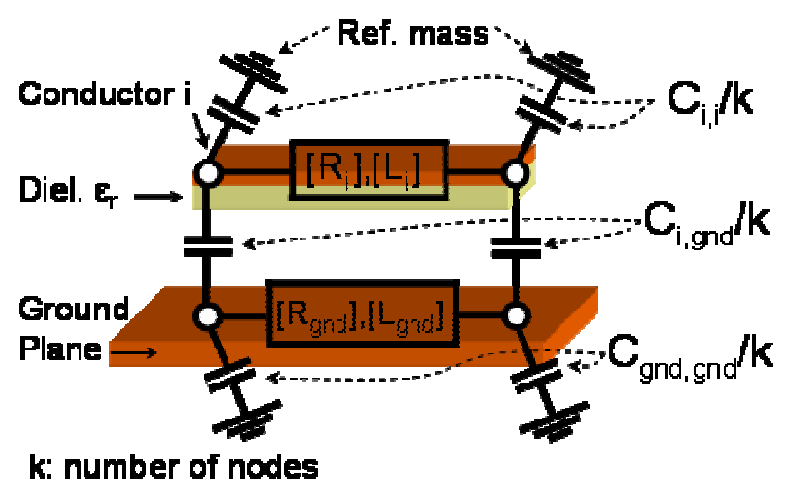

Fig. 4. Implantation of capacitances

Let us note that the ground plane is not the mass reference of the equivalent circuit because the computation of capacitance with the MoM assumes that this one is situated at the infinity. That is why there are self capacitances $\left(\mathrm{C}_{\mathrm{gnd}, \mathrm{gnd}}\right)$ between the ground plane and the mass reference.

Inductive and resistive parts of the layout are calculated with PEEC method. Their equivalent matrix can be extracted and implemented as a macro-block in a circuit simulator. This PEEC model can be then completed with the capacitive circuit computed with the MoM method presented in the previous section. Fig. 5 shows a view of the schematic of a circuit simulator. 


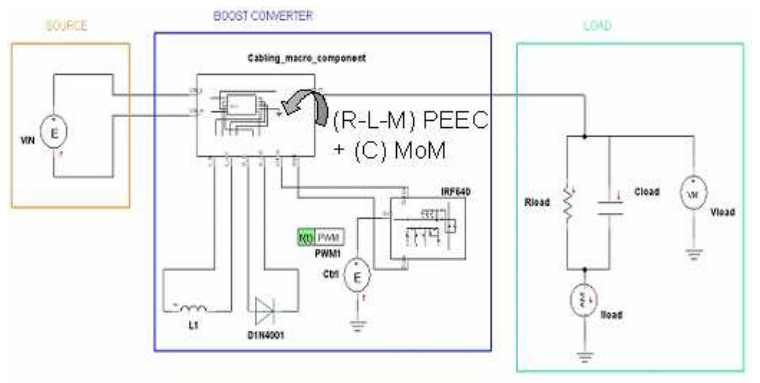

Fig. 5. a- Schematic of a circuit simulator

\section{MODELLING ELECTROMAGNETIC INTERFERENCES}

In order to answer the same conditions of standard measurements, a Line Impedance Stabilization Network (LISN) is added in the circuit simulation. It is then possible to model the common and differential mode currents in the converter. By the way the far radiated emissions of electromagnetic field can be modelled and compared to measurement. One part of those emissions comes from the loop of common mode currents through the stray capacitances and the ground plane.

Fig. 6 presents the application of this method to another boost converter which has been analysed both with measurements and simulations, especially regarding the far electromagnetic field. It has been measured in an anechoic chamber and simulated with MoM combined to PEEC model.
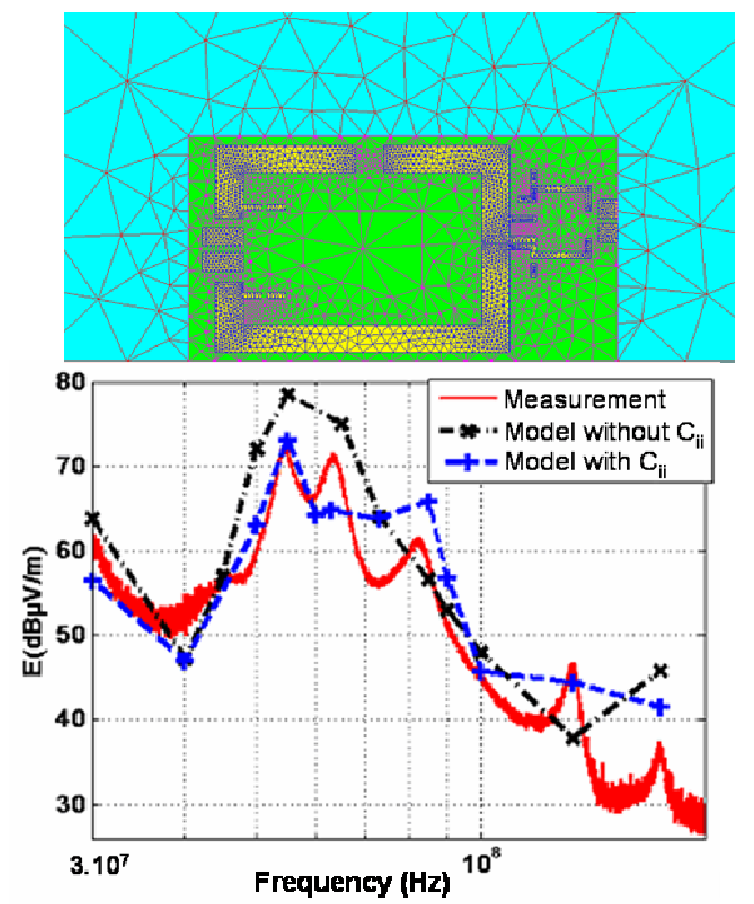

Fig. 6. a- Boost converter mesh, b- Far electric field
Fig. 6a presents the meshing and Fig. $6 \mathrm{~b}$ the magnitude of the electric field measured and calculated to 3 meters from the chopper in a plane perpendicular to the tracks of copper. The results are plotted in the frequency range between 30 and 200 $\mathrm{MHz}$. The most complete model, with $\mathrm{C}_{\mathrm{ii}}$, shows good agreement with the measurements. Most resonances are detected up to $174 \mathrm{MHz}$ (the last peak). Moreover, we can observe the influence of the self capacitances on the precision of the simulation results.

This opens new possibilities for design optimisation regarding EMC conducted or radiated performance. Thanks to PEEC and MoM methods which reduce the computational effort it will be possible to make efficient virtual prototyping by easily testing different technologies.

\section{CONCLUSION}

The parasitic capacitances of a real power electronics structure have been calculated in order to complete the R-L-M model of a static converter obtained with PEEC method. Having a more complete electrical equivalent circuit makes it possible to model EMC performance for a wide frequency range. The radiated electric field of a power electronic converter is evaluated using the presented simulation process and successfully compared to measurements.

\section{REFERENCES}

[1] M. Lionet, R. Prades, X. Brunotte, Y. Le Floch, E. Clavel, J.L. Schanen, J.M. Guichon, "Improving conducted EMI forecasting with accurate layout modeling", IEEE Symposium on Embedded EMC, ESIGELEC, oct. 2007

[2] P.-O. Jeannin, J.-L. Schanen, E. Clavel, "Original cabling conditions to insure balanced current during switching transitions between paralleled semiconductors", IEEE Transactions on Industry Applications, Vol. 38, $\mathrm{N}^{\circ} 1,2002$

[3] J. Aime, J. Roudet, E. Clavel, O. Aouine, C. Labarre, F. Costa, J. Ecrabey, "Prediction and measurement of The magnetic near field of a static converter", IEEE International Symposium on Industrial Electronics, Jun. 2007

[4] InCa3D, Cedrat, www.cedrat.com

[5] C.R. Paul, «Introduction to to Electromagnetic Compatibility» John Wiley \& Sons, 1992

[6] E. Clavel, J. Roudet, J-L. Schanen, "A multiconductor transmission line method to study non-perfect ground planes", IEEE - EMC'99, Zurich, 1999, pp $437-440$

[7] O. Chadebec, J.-L. Coulomb, F. Janet, "A review of Magnetostatic Moment Method", IEEE Transactions on Magnetics, Compumag, Jun. 2005

[8] P. Dular, W. Legros, A. Nicolet, "Coupling of Local and Global Quantities in Various Finite Element Formulations its Application to Electrostatics, Magnetostatics and Magnetodynamics", IEEE Transactions on Magnetics, Vol 34, N5, Sept. 1998

[9] E. Durand, "Tome I - Les distributions", Electrostatique, chp Distributions superficielles, p. 246

[10] E. Durand, "Tome III - Méthodes de calcul diélectriques", Electrostatique, chp - Diélectriques, p. 229

[11] K. Nabors, J. Withe, "Multipole-Accelerated Capacitance for 3-D Structures with Multiple Dielectrics", IEEE Transactions on Circuits and Systems, Vol 39, $\mathrm{N}^{\circ} 11$, November 1992

[12] Flux3D, Cedrat, www.cedrat.com

[13] J. Aime, J. Roudet, C. Vollaire, P. Baudesson, J. Ecrabey, "Layout techniques for reduction of common mode current in static converters", IEEE IAS Annual Meeting Report, Oct. 2006, Vol. 5 p. 2296- 230 